NYU-TH-00/02/01, HEP-MN-1940

\title{
The Power of Brane-Induced Gravity
}

\author{
Gia Dvali ${ }^{*}$, Gregory Gabadadze ${ }^{2 \dagger}$ Marko Kolanović $^{1 \ddagger}$, Francesco Nitti ${ }^{1} \S$ \\ ${ }^{1}$ Department of Physics, New York University, New York, NY 10003 \\ ${ }^{2}$ Theoretical Physics Institute, University of Minnesota, Minneapolis, MN, 55455
}

(May 30, 2018)

\begin{abstract}
We study the role of the brane-induced graviton kinetic term in theories with large extra dimensions. In five dimensions we construct a model with a TeV-scale fundamental Planck mass and a flat extra dimension the size of which can be astronomically large. $4 \mathrm{D}$ gravity on the brane is mediated by a massless zero-mode, whereas the couplings of the heavy Kaluza-Klein modes to ordinary matter are suppressed. The model can manifest itself through the predicted deviations from Einstein theory in long distance precision measurements of the planetary orbits. The bulk states can be a rather exotic form of dark matter, which at sub-solar distances interact via strong $5 \mathrm{D}$ gravitational force. We show that the induced term changes dramatically the phenomenology of sub-millimeter extra dimensions. For instance, high-energy constraints from star cooling or cosmology can be substantially relaxed.
\end{abstract}

\footnotetext{
*e-mail:gd23@nyu.edu

†e-mail: gabadadz@physics.umn.edu

${ }^{\ddagger}$ e-mail: mk679@nyu.edu

§e-mail: fn230@nyu.edu
} 


\section{INTRODUCTION}

It has been known for some time [1.2] that in theories with large extra dimensions the scale at which gravity becomes strong, $M$, can be much lower than the four-dimensional Planck mass $M_{P}$. In this approach the standard hierarchy problem takes a different meaning since the field theory cut-off is lowered. The four-dimensional gravity is weak due to the fact that gravitational flux spreads into $N$ extra dimensions. The relation between fundamental and observed Planck scales takes the form:

$$
M_{P}^{2}=M^{2+N} R^{N},
$$

where $R$ is the circumference of the extra dimensions. In this framework the Standard Model particles are localized on a brane whereas gravity propagates in the bulk of $N$ new dimensions. Until now, it was assumed that if $M \sim$ few $\mathrm{TeV}$ the $N=1$ case is ruled out by observations since it would require $R \sim 10^{16} \mathrm{~cm}$ (the solar system size). As to the higher codimensions, they allow $R$ to be around a millimeter or smaller. The purpose of the present paper is to argue that the framework of compact flat extra dimensions can be dramatically modified due to the presence of the brane-induced kinetic term for higher dimensional graviton [3, [4]

$$
S_{\text {ind }} \propto \int d^{4} x \sqrt{|\operatorname{det} \bar{g}|} \bar{R}(x),
$$

where $\bar{g}$ is the higher dimensional metric evaluated at the position of the brane, and $\bar{R}(x)$ is the corresponding four dimensional Ricci scalar. As a result of this term the flat extra dimension can be astronomically large, and many high energy constraints can be lifted.

In fact, in the approach of [3, [4], the extra dimension was taken to be truly infinite. In spite of this, it was shown that the model reproduces four-dimensional gravity due to the four-dimensional curvature term in the brane worldvolume theory.

To be more precise let us consider 5-dimensional Minkowski space with a standard bulk gravitational action

$$
S_{\text {bulk }}=\int d^{4+1} X \sqrt{|G|} \mathcal{L}\left(G_{A B}, \mathcal{R}_{A B C D}, \Phi\right),
$$

where the capital Latin indices run over $D=(4+1)$-dimensional space-time. $G_{A B}$ denotes the metric of 5 -dimensional space-time, $\mathcal{R}_{A B C D}$ is the 5 -dimensional Riemann tensor and $\Phi$ collectively denotes other fields. Suppose that there is a 3-brane in this space. The 3-brane can be realized as a soliton of the corresponding field equations. We split the coordinates in 5-dimensions as follows:

$$
X^{A}=\left(x^{\mu}, y\right) \text {, }
$$

where Greek indices run over four-dimensional brane worldvolume, $\mu=0,1,2,3$, and $y$ is the coordinate transverse to the brane. In order to reduce our discussion to its main point the brane will be taken to have zero width". In this approximation the brane action takes the form:

\footnotetext{
${ }^{1}$ This is a good approximation when the mass of the field (presumably a scalar) out of which the brane is made is bigger than the energy scale of the low-energy effective theory.
} 


$$
S_{3-\text { brane }}=-T \int d^{4} x \sqrt{|\operatorname{det} \bar{g}|}
$$

where $T$ stands for the brane tension and $\bar{g}_{\mu \nu}=\partial_{\mu} X^{A} \partial_{\nu} X^{B} G_{A B}$ denotes the induced metric on a brane]. In most of this work, unless otherwise stated, we neglect brane fluctuations. Therefore, the induced metric can be written as follows:

$$
\bar{g}_{\mu \nu}(x)=G_{\mu \nu}(x, y=0) .
$$

In general, there could be localized matter fields on the brane worldvolume. These can be taken into account by writing the following action for the brane:

$$
\tilde{S}_{3-\text { brane }}^{\text {matter }}=S_{3-\text { brane }}+\int d^{4} x \sqrt{|\operatorname{det} \bar{g}|} \tilde{\mathcal{L}}(\phi),
$$

where $\phi$ collectively denotes all the localized fields for which the four-dimensional Lagrangian density is $\tilde{\mathcal{L}}$. In the classical theory, which we are discussing so far, the $4 \mathrm{D}$ Ricci scalar on the brane worldvolume is not present. Thus, the localized particles separated at a distance $r$ on a brane interact via the $(4+1)$-dimensional gravitational force-law, that is $F \sim 1 / r^{2+1}$. This holds as long as the classical theory is concerned. However, in the full quantum theory the 4D Ricci scalar will be generated (along with other terms) on the brane worldvolume. This is due to quantum loops of the matter fields which are localized on the brane [3: [1]. As a result, the following worldvolume terms should be included when one considers the full quantum theory:

$$
S_{\text {ind }}=\bar{M}^{2} \int d^{4} x \sqrt{|\operatorname{det} \bar{g}|}\left[\bar{\Lambda}+\bar{R}(x)+\mathcal{O}\left(\bar{R}^{2}\right)\right],
$$

where $\bar{M}^{2} \equiv r_{c} M^{3}$ is some parameter which depends on the details of the worldvolume model [3, [4. $\bar{\Lambda}$ in (1.8) is an induced four-dimensional cosmological constant. The role of this term is to renormalize the brane tension. Furthermore, $\bar{R}(x)$ is the four-dimensional Ricci scalar which is constructed out of the induced metric $\bar{g}_{\mu \nu}(x)$ defined in (1.6). In five-dimensional Minkowski space a brane with nonzero tension inflates [0, 6]. Therefore, to avoid the worldvolume inflation we fine tune the brane tension $T$ and the brane worldvolume cosmological constant $\bar{\Lambda}$ so that the net tension is vanishing

$$
T^{\prime} \equiv T-\bar{\Lambda} \bar{M}^{2}=0 .
$$

This is a usual fine tuning of the four-dimensional cosmological constant.

The graviton propagator resulting from such a system is quite peculiar. Ignoring the tensor structure for a moment we obtain for the corresponding Green's function the following expression:

$$
\tilde{G}_{R}(p, y)=\frac{1}{2 M^{3} p+\bar{M}^{2} p^{2}} \exp \{-p|y|\}
$$

\footnotetext{
${ }^{2}$ Below we will imply that the Gibbons-Hawking surface term is also present on the worldvolume so that it provides the correct bulk Einstein equations.
} 
Here $p^{2}$ is a four-dimensional Euclidean momentum and $p \equiv \sqrt{p^{2}}$. For sources which are localized on the brane $(y=0)$, this propagator reduces to a massless four-dimensional Green's function

$$
\tilde{G}_{R}(p, y=0) \propto \frac{1}{p^{2}}
$$

provided that $p \gg 1 / r_{c}$. Thus, at distances $r \ll r_{c}$ we observe the correct Newtonian behavior of the potential

$$
V(r) \propto \frac{m_{1} m_{2}}{r \bar{M}^{2}}
$$

At large distances, $r \gg r_{c}$, however, the behavior of the Green's function changes

$$
\tilde{G}_{R}(p, y=0) \propto \frac{1}{p} .
$$

This gives rise to a Newtonian potential which scales in accordance with the laws of a five-dimensional theory 3

$$
V(r) \propto \frac{1}{r^{2} M^{3}}
$$

This somewhat puzzling behavior can be understood in two equivalent ways which we briefly discuss. First let us adopt the five-dimensional point of view. In this language, although there is no localized massless particle, there exists a localized resonance state in the spectrum. The lifetime of this resonance is $\sim r_{c}$. The resonance decays into the continuum of modes. This can be manifestly seen using the Källen-Lehmann representation for the Green's function

$$
\tilde{G}_{R}(p, y=0)=\frac{1}{2 M^{3} p+M^{3} r_{c} p^{2}}=\int_{0}^{\infty} \frac{\rho(s) d s}{s+p^{2}},
$$

where the spectral density as a function of the Mandelstam variable $s$ takes the form:

$$
\rho(s) \propto \frac{1}{\sqrt{s}} \frac{r_{c}}{4+s r_{c}^{2}}
$$

As $r_{c} \rightarrow \infty$ the spectral density tends to the Dirac function $\rho(s) \rightarrow$ const. $\delta(s)$ describing a stable massless graviton (this corresponds to the limit when the bulk kinetic term can be neglected). Summarizing, at distances $r<r_{c}$ the resonance mimics the massless exchange, and therefore mediates the $1 / r^{2}$ force. At larger distances, however, it decays into the continuum, and as a result the force-law becomes that of a five-dimensional theory, $\sim 1 / r^{3}$.

3 Transition to five dimensional regime at long distances is also a characteristic feature of the models with "quasi-localized" gravity [0 13]. However, these models usually include negative norm states 911 . 
A different but equivalent way to understand the above result is to adopt the point of view of the four-dimensional mode expansion. The analysis of the linearized equation for the small fluctuations shows (see Appendix) that there is a continuum of 4D massive states with wave-function profiles that, at the location of the brane, are suppressed by the following factor

$$
\left|\phi_{m}(y=0)\right|^{2}=\frac{1}{4+m^{2} r_{c}^{2}} .
$$

The Newtonian force on the brane is mediated by the exchange of all these Kaluza-Klein (KK) modes. These give rise to the expression:

$$
V(r) \propto \frac{1}{M^{3}} \int_{0}^{\infty} \frac{d m}{4+m^{2} r_{c}^{2}} \frac{e^{-m r}}{r} .
$$

At any distance $r$ the dominant contribution comes from the modes lighter than $m=1 / r$. The modes with $m<1 / r_{c}$ have unsuppressed wave-functions on the brane. Therefore, for $r>r_{c}$ the interaction picture is similar to that of a five-dimensional theory. In contrast, when $r<r_{c}$ the picture changes since the modes with $m>1 / r_{c}$ have suppressed couplings. Although the number of the modes which participate in the exchange at a given distance $r<r_{c}$ is the same as in the five-dimensional picture, their contributions are suppressed. Thus, the number of the light modes effectively contributing to the exchange "freezes-out" and the resulting behavior of the potential is $1 / r$.

As pointed out in [3], this model exhibits the van Dam-Veltman-Zakharov (vDVZ) discontinuity [14, 15] in the tensor structure of the graviton propagator in the lowest tree-level approximation (as would any flat space ghost-free theory where $4 D$ gravity is mimicked by the exchange of (a continuum of) massive spin-2 particles [9]). Another disadvantage of this

model is the low value of the crossover scale $r_{c} \simeq 10^{16} \mathrm{~cm}$. This would imply that Newtonian gravity is modified at this distance, which is certainly unacceptable.

In the present paper we show that both aforementioned difficulties are circumvented if the fifth dimension is compactified on a circle of macroscopic size $R$. We will argue that $R$ can be of astronomical size. In this framework, the four-dimensional Planck scale emerges as

$$
M_{P}^{2}=M^{3}\left(R+r_{c}\right) \text {. }
$$

Gravity on the brane is dominated by a lowest lying massless KK zero mode, which has an unsuppressed wave-function. As a result there is no vDVZ discontinuity problem. Contributions from the massive KK modes give rise to small corrections to Newtonian gravity. These corrections are mostly constrained by the data on the motion of the inner planets, Moon and satellite experiments. We study constraints coming both from astronomy (planetary motion) and from astrophysics (star cooling), and show that the model is consistent with all the observations.

\section{THE MODEL}

We would like to consider a simplest possible model with a single brane embedded in five-dimensional space with one compact dimension. The action of our model takes the form: 


$$
S=M^{3} \int d^{4} x \int_{0}^{R} d y \sqrt{G} \mathcal{R}_{(5)}+M^{3} r_{c} \int d^{4} x \sqrt{|\bar{g}|} \bar{R}
$$

As we mentioned before, the four-dimensional Planck mass is set by the sum of the two distance scales $r_{c}$ and $R$ through the relation (1.19). For the solution of the hierarchy problem along the lines of Ref. [1] we can put $M \lesssim \mathrm{TeV}$. This sets the largest of the two scales, $R$ and $r_{c}$, to be larger then $\sim 10^{16} \mathrm{~cm}$ or so. It is tempting to assume that the origins of these two scales are related. Some possible ideas along this direction will be discussed later. For the time being we shall take them as the parameters of the theory.

We are interested in the Newtonian gravitational interaction of the brane-localized sources. In this section we will ignore the tensor structure of the graviton propagator and concentrate on its scalar part. A straightforward calculation (see Appendix) leads to the following result for the Euclidean two-point Green's function:

$$
\Delta(p, 0)=\frac{1}{p^{2}}\left[\frac{1}{1+\left(1 / p r_{c}\right) 2 \tanh (p R / 2)}\right] .
$$

For $r_{c} \gg R$ this propagator exhibits the $1 / p^{2}$ - behavior both for large and for small momenta.

$$
\begin{array}{ll}
\Delta(p, 0) \simeq \frac{1}{p^{2}}\left[1-\frac{2}{p r_{c}}\right] & p R \gg 1 \text { (short distances) }, \\
\Delta(p, 0) \simeq \frac{1}{p^{2}}\left[1-\frac{R}{r_{c}}\right] & p R \ll 1 \text { (large distances) } .
\end{array}
$$

Therefore the potential is four-dimensional both for large and for small distances. Note that the maximal deviation in the coefficient of $1 / p^{2}$ is of order $R / r_{c}$.

Let us now turn to Minkowski space-time by rotating $p \rightarrow i p_{M}$. The propagator takes the form:

$$
\Delta\left(p_{M}, 0\right)=-\frac{1}{p_{M}^{2}}\left[\frac{1}{1+\left(1 / p_{M} r_{c}\right) 2 \tan \left(p_{M} R / 2\right)}\right] .
$$

This expression has poles at $r_{c} p_{M}=-2 \tan \left(p_{M} R / 2\right)$. In order to understand better this result let us turn to the KK expansion. The mode decomposition is given in Appendix. As one would expect, there is a discrete tower of KK states. For $r_{c} \ll R$ the KK masses approach the usual KK spectrum, $m_{n}=2 \pi n / R$. For $r_{c} \gg R$ all modes (except the zero mode) tend to the asymptotes of the tangent function: $m_{0}=0, m_{n} \simeq(2 n-1) \pi / R$. This is in agreement with the pole structure of the propagator (2.4). Note that the level spacing for fixed $R$ does not change. For arbitrary $r_{c}$ the mass of the $n$ 'th state is in the interval $((2 n-1) \pi / R, 2 n \pi / R)$.

Let us calculate the static potential between two objects on the brane which are separated by a distance $r$. For this we perform the KK decomposition of the corresponding 5D fields. This is done in Appendix. The result can be summarized as follows:

$$
V(r) \simeq \frac{G_{0}}{r}+\sum_{n=1}^{\infty} G_{n} \frac{e^{-m_{n} r}}{r},
$$

where for the case $r_{c} \gg R$ we can use $m_{n} \simeq(2 n-1) \pi / R$ and after some simplifications we find (see Appendix): 


$$
\begin{aligned}
G_{0} & =\frac{1}{M^{3}} \frac{1}{r_{c}+R} \simeq \frac{1}{r_{c} M^{3}} \equiv G_{\infty} \\
G_{n} & =\frac{1}{r_{c} M^{3}}\left(\frac{R}{r_{c}}\right) \frac{2}{[(2 n-1) \pi / 2]^{2}+R / r_{c}+\left(R / r_{c}\right)^{2}} \\
& \simeq \frac{1}{r_{c} M^{3}}\left(\frac{2}{\pi^{2}}\right)\left(\frac{R}{r_{c}} \frac{1}{n^{2}}\right) .
\end{aligned}
$$

Finally, we derive the potential:

$$
V(r) \simeq \frac{G_{\infty}}{r}\left(1+\frac{2}{\pi^{2}} \frac{R}{r_{c}} \sum_{n=1}^{\infty} \frac{e^{-(2 n-1) \pi r / R}}{n^{2}}\right) .
$$

The term in the parenthesis in this expression changes slowly with $r$ and the magnitude of the change is of the order $R / r_{c}$. One could think of (2.8) as being the Newton potential with an effective space-dependent Newton's constant. To estimate the variation of the effective gravitational constant we evaluate the difference of the potentials at two distinct points. For a given distance $r$ in the KK sum we can neglect the modes with $n>R / r$ (since these are exponentially suppressed) and replace the exponential by 1 in the remaining terms for which $n<R / r$. The variation of the Newton constant for two points $r_{1}$ and $r_{2}\left(r_{1}>r_{2}\right)$ evaluates as follows:

$$
\frac{G\left(r_{1}\right)-G\left(r_{2}\right)}{G_{\infty}} \sim \frac{R}{r_{c}} \sum_{n=R / r_{1}}^{R / r_{2}} \frac{1}{n^{2}}
$$

Moreover, the potential can be evaluated exactly and the expression can be expanded for small and large values of $r / R$. For $r / R \ll 1$ and $R \ll r_{c}$, we find:

$$
V(r) \simeq \frac{G_{\infty}}{r}\left[\frac{1}{1+R / r_{c}}+\frac{R}{r_{c}}\left(1+\frac{4}{\pi} \frac{r}{R}\left(\ln \frac{r}{R}+C\right)+O\left(\left(R / r_{c}\right)^{2},(r / R)^{2}\right)\right)\right],
$$

where $C=\ln (\pi / 2 e) \simeq-0.54$. Likewise, for $r / R \gg 1$ and $R \ll r_{c}$, the potential reads as follows:

$$
V(r) \simeq \frac{G_{\infty}}{r}\left[\frac{1}{1+R / r_{c}}+\left(\frac{4}{\pi^{2}} \frac{R}{r_{c}}+O\left(\left(R / r_{c}\right)^{2}\right)\right) e^{-\pi r / R}+\ldots\right]
$$

The overall relative change in Newton's constant for $r$ in the interval $[0, \infty]$ is the ratio $R / r_{c}$.

\section{CONSTRAINTS}

\section{A. Tensor Structure of the Propagator}

In the present model $4 \mathrm{D}$-gravity on the brane is mediated by the zero mode graviton with an admixture of the tower of massive KK states. The latter contribution is suppressed

both by the parameter $\alpha \equiv R / r_{c}$ and by the KK number $n^{2}$. In order to determine the tensor structure of the graviton propagator let us compare how it changes when one turns 
from a massless graviton to massive one. In 4D space the momentum-independent part of the tensor structure takes the form:

$$
\frac{1}{2}\left(\eta^{\mu \alpha} \eta^{\nu \beta}+\eta^{\mu \beta} \eta^{\nu \alpha}\right)-\frac{1}{2} \eta^{\mu \nu} \eta^{\alpha \beta}
$$

This can be seen by using, for instance, the harmonic gauge for the fluctuations of the massless gravitational field:

$$
\partial^{\mu} h_{\mu \nu}^{(0)}=\frac{1}{2} \partial_{\nu} h_{\beta}^{(0) \beta}
$$

On the other hand, for a massive graviton one finds:

$$
\frac{1}{2}\left(\eta^{\mu \alpha} \eta^{\nu \beta}+\eta^{\mu \beta} \eta^{\nu \alpha}\right)-\frac{1}{3} \eta^{\mu \nu} \eta^{\alpha \beta}
$$

In the latter case, the equations of motion for a massive graviton (we use the Pauli-Fierz mass term) give rise to the following relation for the fluctuations:

$$
\partial^{\mu} h_{\mu \nu}^{(m)}=\partial_{\nu} h_{\beta}^{(m) \beta}
$$

Using this condition in the Einstein equations naturally leads to the tensor structure given in (3.3) Let us now see what happens in the 5D model. Before compactification one can choose the harmonic gauge in the bulk space:

$$
\partial^{A} h_{A B}=\frac{1}{2} \partial_{B} h_{C}^{C}
$$

This leads to the tensor structure of the form (3.3). Let us consider what happens upon compactification. For the massive KK modes, $m \neq 0$, the $\{\mu \nu\}$ components of the gauge condition (3.5) will turn into the following expression:

$$
\partial^{\mu} h_{\mu \nu}^{(m)}=\frac{1}{2}\left(\partial_{\nu} h_{\beta}^{(m) \beta}+\partial_{\nu} h_{5}^{(m) 5}\right)
$$

while the $\{55\}$ component takes the form:

$$
h_{\mu}^{(m) \mu}(x)=h_{5}^{(m) 5}(x) .
$$

Substituting (3.7) into (3.6) we derive (3.4). Using this, we obtain the following expression for the tensor structure of massive KK gravitons:

$$
\tilde{h}_{\mu \nu}^{(n)}(p, 0) \tilde{T}^{\prime \mu \nu} \propto\left\{\tilde{T}_{\mu \nu} \tilde{T}^{\prime \mu \nu}-\frac{1}{3} \tilde{T}_{\alpha}^{\alpha} \tilde{T}_{\beta}^{\prime \beta}\right\}
$$

\footnotetext{
${ }^{4}$ Note that the condition (3.4) would be an inconsistent gauge choice for the massless gravitons. This is in contrast with the vector field case, where the equation of motion for a massive vector field $A_{\mu}$ (Proca field) would require the relation $\partial^{\mu} A_{\mu}=0$ and this latter is also an acceptable gauge choice for a massless gauge field.
} 
where $\tilde{T}_{\mu \nu}, \tilde{T}^{\prime \mu \nu}$ are Fourier transformed matter source energy-momentum tensors. Therefore in the approximation $R / r_{c} \ll 1$ the expression for the propagator takes the form (the tensor structure of the zero-mode is that of a $4 \mathrm{D}$ theory):

$$
\begin{aligned}
& G_{4}^{\mu \nu \alpha \beta}(p) \simeq G_{\infty}\left(\left(\frac{1}{2}\left(\eta^{\mu \alpha} \eta^{\nu \beta}+\eta^{\mu \beta} \eta^{\nu \alpha}\right)-\frac{1}{2} \eta^{\mu \nu} \eta^{\alpha \beta}\right) \frac{1}{p^{2}}+\right. \\
& \left.\frac{2}{\pi^{2}} \frac{R}{r_{c}} \sum_{n=1}^{\infty} \frac{1}{n^{2}}\left(\frac{1}{2}\left(\tilde{\eta}^{\mu \alpha} \tilde{\eta}^{\nu \beta}+\tilde{\eta}^{\mu \beta} \tilde{\eta}^{\nu \alpha}\right)-\frac{1}{3} \tilde{\eta}^{\mu \nu} \tilde{\eta}^{\alpha \beta}\right) \frac{1}{p^{2}+m_{n}^{2}}\right)
\end{aligned}
$$

where

$$
\tilde{\eta}_{\mu \nu} \equiv \eta_{\mu \nu}+\frac{p_{\mu} p_{\nu}}{m_{n}^{2}}
$$

Thus, the lowest massive KK modes are suppressed by the ratio $R / r_{c} \equiv \alpha$. These contributions might be important. Indeed, as shown in [9] any ghost-free theory in which massive spin-2 KK contribution to 4D gravity is essential should suffer from vDVZ-discontinuity problem [14, 15]. The reason is that in flat space any massive spin- 2 states have 5 polarizations. Three of these polarizations couple to the conserved stress energy tensor with the same strength and contribute to the gravitational potential. This induces a finite deviation from the predictions of Einstein gravity for arbitrarily small mass [14, 15]. Therefore, theories with massive graviton exchange encounter phenomenological difficulties and are severely constraint. We shall try to quantify the implications of this constraint in our model.

As we discussed above, the massive modes give rise to the distinctive tensor structure in the propagator which alters the predictions for light bending by Sun and the precession of Mercury perihelion. Therefore, the massive KK modes must be adequately suppressed. The sum in (3.9) is rapidly convergent. As a result, the dominant contribution comes from a few lightest KK modes. Using the present experimental data [16], this gives the following constraint:

$$
\alpha \equiv \frac{R}{r_{c}}<3 \times 10^{-4}
$$

\section{B. Planetary Motion}

We shall now examine the constraints on the size of the extra dimension $R$, coming from solar system dynamics. An object orbiting in the gravitational field will exchange a certain number of the massive KK modes. This is determined by the distance of the object from the center of the potential. For example, Pluto will effectively exchange less KK modes than Mercury. This will be seen as an effective change of Newton's constant and will constraint the possible values of $R$. In the present model, the total change in Newton's constant felt by an object falling from infinity towards the center of the potential would be $R / r_{c}$. From the expansions of the potential (2.10) and (2.11) it is obvious that most of the change happens in the range $0<r<R$.

Experimental bounds on the spatial variation of Newton's constant over planetary distances are quite strong. They come from precise tracking of planetary orbits with the help 
of radar beams and space probes. The results of those measurements are presented in the work [17] (an updated and detailed discussion can be found in [18]). The modification of the Newton's potential assumed in [17] is

$$
\Delta V(r)=\alpha G_{\infty} \frac{e^{-r / \lambda}}{r}
$$

where $\alpha$ and $\lambda$ are parameters that describe deviations from standard potential. A nonzero value of these parameters would result in deviations from the Kepler's third law and would induce anomalous precessions of the planets' perihelia. The precise measurements of orbits of the inner planets and satellites put bounds on the values of $\alpha$ and $\lambda$ that are summarized in Ref. 17,18.

Let us relate approximately the parameters of our model with the parameters in (3.12). For simplicity we will use the following estimate

$$
\begin{aligned}
\Delta V(r) & \simeq \frac{G_{\infty}}{r}\left(\frac{2}{\pi^{2}} \frac{R}{r_{c}} \sum_{n=1}^{\infty} \frac{e^{-(2 n-1) \pi r / R}}{n^{2}}\right)=\frac{2 R}{\pi^{2} r_{c}} G_{\infty} \frac{e^{-r \pi / R}}{r}+\frac{2}{\pi^{2}} \frac{R}{r_{c}} \sum_{n=2}^{\infty} \frac{e^{-(2 n-1) \pi r / R}}{n^{2}} \\
& <\frac{2 R}{\pi^{2} r_{c}} G_{\infty} \frac{e^{-\pi r / R}}{r}\left(1+\sum_{n=2}^{\infty} \frac{1}{n^{2}}\right)=\frac{R}{3 r_{c}} G_{\infty} \frac{e^{-\pi r / R}}{r} .
\end{aligned}
$$

With this estimate we can relate the parameters of our model with the parameters in (3.12)

$$
\alpha \sim \frac{R}{r_{c}}, \quad \lambda \sim R
$$

The bound on $\alpha$ is very stringent $\left(\alpha \leq 10^{-9}\right.$, [17, 18]) for the values of $\lambda$ in the range from the Earth radius to the Solar System size. If we assume $M \sim \mathrm{TeV}$ we conclude that the size of extra dimension $R$ must be either smaller than the Earth radius, or bigger than the solar system size.

Let us discuss these two possibilities separately. We start with the case when $R$ is larger then the solar system size. In this case, the estimate (3.14) is actually not the most precise one. The bounds on $\alpha$ and $\lambda$ shown in [17 were derived using the spatial variation of Newton's constant that appears in the force, rather than in the potential. We can compare the correction to the force in our model and the same correction for the pure Yukawa type modification, both shown in Fig.1. We see that the force correction in our model is a much steeper function on small values of $r / R$.

The measured quantity is the parameter $\eta_{p}$ that describes deviations from third Kepler's law. The ratio of semi-major axis can be measured $\left(a_{p}^{\text {meas }}\right)$ and compared to prediction of Kepler's law $\left(a_{p}\right)$. The following equation relates $\eta$ and the value of Newton's constant at the Earth $\left(G_{E}\right)$ and at the planet's orbit $\left(G_{p}\right)$.

$$
\frac{a_{p}}{a_{p}^{\text {meas }}}=1+\eta_{p}=\left(\frac{G_{p} M_{s}}{G_{E} M_{s}}\right)^{1 / 3} .
$$

Newton's constant is the one that appears in the force law, and $M_{s}$ is the mass of the Sun. The bound on the parameters $\eta_{p}$ is of the order $10^{-9}-10^{-10}$ for the inner planets. 


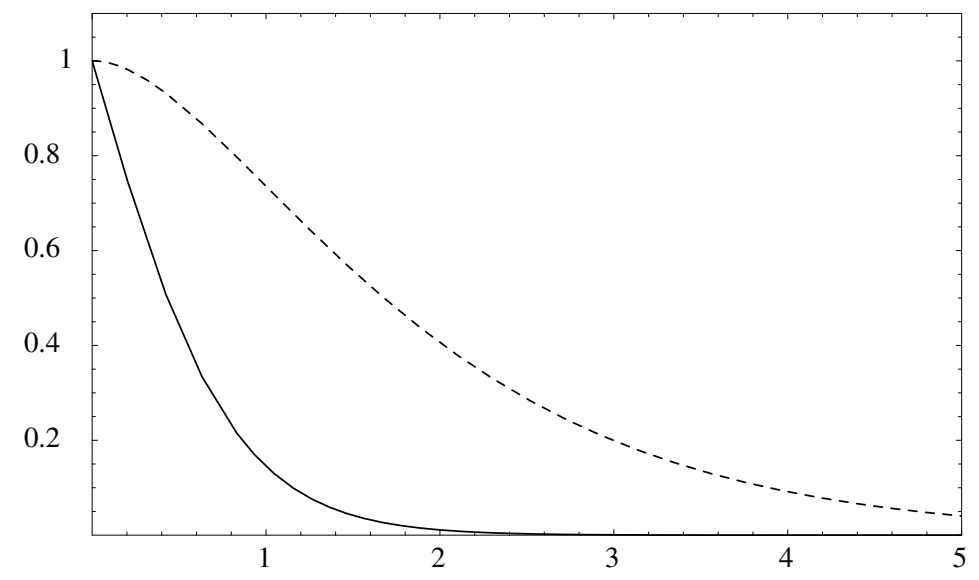

Figure 1: The correction to Newton's force for our model (solid line, $R / r_{c}=10^{-4}$ ) and for the Yukawa type correction (dashed line, $\alpha=10^{-4}$ ). The relative correction (normalized to the strength of Newton's force) is given is units of $10^{-4}$. The $x$ axis measures $r / R$ for our model and $r / \lambda$ for the Yukawa correction.

The most precise bound on the spatial variation of Newton's constant $\left(\eta_{p}\right)$ comes from the relation between the Earth and inner planets, i.e., in the regime of small $r / R$, for $R$ greater then the solar system size. In that regime, the change in force will be bigger for our model than for the Yukawa type correction and we have to compensate that effect by choosing somewhat larger values of $R$ and $r_{c}$. Numerical analysis shows that with the choice of $R \approx 10^{16} \mathrm{~m}$, and $R / r_{c} \approx 10^{-4}$, the bounds can be safely satisfied. Since the Planck scale is roughly given by $r_{c} M^{3}$, an increase of $r_{c}$ by some factor would lower the fundamental Planck scale for a much milder value of a cubic root. With the new bound of $R>10^{16} \mathrm{~m}$ and $R / r_{c}>10^{-4}$ the fundamental Planck scale is pushed to approximately $100 \mathrm{GeV}$.

Similarly, we calculated the contribution to the anomalous precession of Mercury and Mars in our model, and we found that it can be within the accepted values if the above limits on $R$ and $R / r_{c}$ are satisfied

The second possibility is to take the size of the extra dimension to be anywhere less than the Earth radius $\left(\sim 10^{7} \mathrm{~m}\right)$, in order to satisfy constraints from Earth-Moon and artificial satellite experiments. This "regime" is much safer and the bounds are very weak, as can be seen in [17,18]. In the case $R \sim 10^{7} \mathrm{~m}, r_{c}$ can range form $\sim 10^{11} \mathrm{~m}$ to $\sim 10^{19} \mathrm{~m}$, corresponding to a fundamental Plank scale of $\sim 10 \mathrm{TeV}$ down to $100 \mathrm{GeV}$, respectively. Of course the value of $R$ can be lowered at will, down to $1 / M$, while keeping $r_{c}$ fixed in order to generate the correct value for the four dimensional Plank scale from the 'low' fundamental scale $\sim 1 \mathrm{TeV}$. To point out the importance of our result in this regime, we stress that our model makes possible the existence of a single extra dimension of very large size without contradiction with present observations, while keeping the value of the fundamental Plank scale around the $\mathrm{TeV}$.

The mechanism of suppression of heavy modes in our framework can relax the high energy constrains on the models with submillimeter extra dimensions.

To summarize, there are two windows of the parameter space in the present model. The first one, in which the extra dimension has astronomic scale, is strongly constrained by the solar system observations which force $R \geq 10^{16} \mathrm{~m}$ (for $R / r_{c} \simeq 10^{-4}$ ). In the second scenario 
$R$ can be anywhere below the Earth radius and $1 / M$.

\section{Star Cooling}

The most severe constraint on theories with low scale gravity comes from astrophysics since a stellar object can cool by radiating bulk gravitons [2]. Let us estimate the rate of star cooling in the present case. Let the temperature inside the star be $T$. Then only the gravitons with masses $m_{n}<T$ are produced efficiently. The rate of emission of the $n$th KK graviton is given by

$$
\Gamma_{n} \sim T^{3} G_{n} .
$$

We have to sum over all states up to $n \sim T R$. This gives

$$
\Gamma_{\text {total }}(T) \sim \frac{2 T^{3} R}{\pi^{2} M^{3} r_{c}^{2}} \sum_{1}^{R T} \frac{1}{n^{2}} \simeq \frac{T^{3} R}{3 M^{3} r_{c}^{2}} \simeq \frac{\alpha}{3} \frac{T^{3}}{M_{P}^{2}} .
$$

This is less than the rate of just zero mode graviton production, and is totally negligible. Note that the contribution coming from the emission of a pair of KK gravitons via the virtual zero mode graviton exchange is of the same order, and is practically insensitive to the value of $R$ and $M$. To see this let us consider a scattering process on the brane with a virtual zero mode graviton emission which subsequently decays into a pair of KK gravitons. The example of such a process can be a gravi-brehmstrahlung process, an electron scattering in the field of a nucleus and radiating gravitons. Let the typical energy in the process be $E$. For definiteness we take $E>M$, in order to put the strongest constraint. The rate of the process then becomes

$$
\Gamma \sim \frac{E^{3} M^{2}}{M_{P}^{4}}(M R) \sim \alpha \frac{E^{3}}{M_{P}^{2}},
$$

where we have cut-off graviton momenta by $M$. Surprisingly, this rate is similar to the one of single KK production, due to the suppression of direct KK production rate. Most importantly it has no dependence on either $R$ or $M$. Thus the KK emission in high-energy processes a priory places no bound on either of this parameters. However, we do not expect $M$ to be smaller than the inverse mm scale, due to no observed deviation from Newtonian gravity on larger distances, which gives the upper limit on the size of extra dimension $R \sim \alpha 10^{63} \mathrm{~cm}$. Thus the star cooling places no constraint on our scenario. It is useful to contrast this with the scenario of [1]. There, the dominant contribution to the cooling process comes from the production of heaviest modes, due to their high number. In the present case however, the wave functions of the heavy modes are suppressed on the brane and therefore cannot be produced efficiently. This is a generic property of the given framework with the induced kinetic term: in a high-energy process on the brane, the lightest KK states are the ones produced most efficiently. The extra dimension is more transparent for the softer modes! 


\section{Cosmology}

In this section we will consider cosmological constraints coming from the overproduction of bulk states. Not surprisingly, in analogy with star cooling, the cosmological constraints turn out to be rather mild. In order to be as model independent as possible, we shall discuss the following initial conditions for the hot big bang:

1) The bulk is virtually empty;

2) The brane states are in thermal equilibrium at some temperature $T_{\text {brane }}$, which can not be higher than the "normalcy" temperature $T_{*}$.

The normalcy temperature is defined as the temperature below which the Universe expands as being effectively four-dimensional. This requirement then automatically restricts $T_{\text {brane }}<T_{*}$. Obviously, we would like $T_{*}$ to come out at least as high as the nucleosynthesis temperature. As we shall see below, $T_{*}$ could be much higher, even as high as the cut-off scale $\left(T_{*} \sim M\right)$. This fact allows for the standard nucleosynthesis scenario to proceed unaffected, and also to implement one of the conventional baryogenesis mechanism, for generating the baryon asymmetry of the Universe.

As discussed in [2], in theories with large extra dimensions the overproduction of bulk KK states can alter the standard cosmological expansion in two different ways. First, the energy density on the brane changes due to the "evaporation" into the bulk states. Secondly, the produced bulk states may dominate the energy density and over-close the universe. Let us consider constraints coming from these two effects separately.

\section{Cooling by Evaporation into Bulk States}

As estimated above for star cooling at temperature $T$ the evaporation rate into the bulk gravitons is given by (3.17). The resulting change of the matter energy density on the brane due to evaporation is given by

$$
\left.\frac{d \rho}{d t}\right|_{\text {evaporation }} \sim-T^{4} \Gamma_{\text {total }}(T) \sim-\frac{T^{7}}{M_{P}^{2}} \alpha .
$$

Note that this is by a factor $\alpha$ smaller than the cooling rate due to the production of the standard zero-mode graviton, and is totally negligible in comparison to the cooling rate caused by the cosmological expansion

$$
\left.\frac{d \rho}{d t}\right|_{\text {expansion }} \sim-3 H \rho \sim-3 \frac{T^{2}}{M_{P}} \rho,
$$

where $H$ is the Hubble parameter. For instance, in the radiation dominated epoch $(H \sim$ $\left.T^{2} / M_{P}\right)$, the ratio of the two rates is

$$
\frac{\left.\frac{d \rho}{d t}\right|_{\text {evaporation }}}{\left.\frac{d \rho}{d t}\right|_{\text {expansion }}} \sim \frac{T}{M_{P}} \alpha .
$$

This is a very small number even for $T \sim \mathrm{TeV}$. The reason for this suppression can again be understood from the 'infrared transparency' of the theory: since the heavier gravitons' wave-functions are strongly suppressed on the brane, their production is not efficient enough to affect the brane-cooling process. 


\section{Overclosure by Gravitons}

Due to their suppressed couplings, KK gravitons are harder to produce on the brane. Moreover, due to the same suppression the KK modes are more long-lived as well. Thus, we have to examine constraints coming from their possible overproduction.

The lifetime of a "cold" KK graviton of mass $m$ can be written as follows:

$$
\tau_{m} \sim \frac{M_{P}^{2}\left(r_{c} R\right)}{m}
$$

This turns into the following relation upon using (1.19)

$$
\tau_{m} \sim\left(\frac{M_{P}}{M}\right)^{6} \frac{\alpha}{m}
$$

Thus, even the gravitons as heavy as $m \sim M$ are stable for all the practical purposes and can over-close the universe if produced with a sufficiently large number density. This puts some bound on $T_{*}$ which we shall estimate below. Assuming that all the produced KK gravitons are stable and do not decay back to the brane, the energy density which is "pumped" into the bulk due to the brane-evaporation (in the process of the normal expansion) is

$$
\delta \rho=\left.\int_{t_{i n}}^{t e q} \frac{d \rho}{d t}\right|_{\text {evaporation }} d t \sim \int_{T_{e q}}^{T_{*}} \frac{T^{4}}{M_{P}} \alpha d T \sim \frac{T_{*}^{5}}{M_{P}} \alpha .
$$

Here $T_{e q} \sim \mathrm{eV}$, is the standard crossover temperature and we neglected the later period of matter domination as well as the order one factors in the integral. Most of the energy density gets pumped in the bulk during one Hubble time after $t=t_{i n}$. Thus, soon after the initial time the bulk becomes populated by KK states with the energy density given by (3.24). Most of these particles are relativistic and their energy density will redshift as radiation $\left(\rho \sim T^{4}\right)$. However, to make our bound most conservative let us assume that most of the energy density is stored in the cold KK modes that redshift as matter $\left(\rho \sim T^{3}\right)$. Even in this case their energy density at the time of standard crossover will be given by

$$
\rho_{K K} \sim \frac{T_{*}^{2}}{M_{P}} \alpha T_{e q}^{3}
$$

Requiring that this be much smaller than the energy stored in the usual matter (at the same time $\left.t=t_{e q}\right)$, which, by definition, is $\rho_{\text {matter }} \sim T_{e q}^{4} \sim(10 \mathrm{eV})^{4}$, we get the following bound on $T_{*}$

$$
T_{*}^{2}<\frac{M_{P}}{\alpha} e V=\frac{10^{10}}{\alpha} \mathrm{GeV}^{2}
$$

This bound can be easily satisfied even for $T_{*} \sim M$.

In conclusion, we see that in contrast with the scenario of [1] there are no essential cosmological constraints from overproduction of bulk states due to the brane cooling. We have to stress, however, that this analysis can not capture more model dependent possibilities.

For instance, if the KK states are produced by some other means in the early universe, they could either over-close the Universe or serve as unusual (and interesting) dark matter 
candidates. To avoid the overproduction we have to assume that there was a period of the inflation that diluted the bulk and reheated only the brane 9 .

\section{ON THE ORIGIN OF THE CROSSOVER SCALE}

In this section we shall discuss a possible origin of the large distance scale $r_{c}$. The goal is to explain the large coefficient in front of the four-dimensional curvature term in Eq. (1.8). As already noted, in the effective field theory picture this term is not constrained by any symmetry and can emerge with an a priory unrestricted coefficient. However, it is desirable to have better understanding of this issue. As suggested in [3, 4], this term is induced due to quantum loops of the states which are localized on the brane and which interact with high-dimensional gravity. The resulting strength depends on the number of such states as well as their masses [4]. Thus, the large mass hierarchy could be obtained due to the large multiplicity of states propagating in matter loops on the brane. However, there can be other effects that may significantly contribute to the magnitude of this term. One possibility is to consider a brane-scalar field $\xi$ which is non-minimally coupled to gravity

$$
S_{\text {brane }}=M^{2} \int d^{4} x \sqrt{|\operatorname{det} \bar{g}|} \exp \left(\frac{\xi}{M}\right) \bar{R}(x) .
$$

One could assume that, due to some dynamics, $\xi$ develops a vacuum expectation value close to the scale $M$. This is in no contradiction with any fundamental principle, as long as $\xi$ is somewhat lighter than $M$. Shifting the field $\xi \rightarrow<\xi>+\xi$ we end up with an exponentially large scale $r_{c}=M^{-1} \mathrm{e}^{\frac{<\xi>}{\mathrm{M}}}$. Note that in the shifted vacuum the theory is consistent up to the energies of order $\sim M$. Moreover, the perturbative treatment is valid since the emission of $\xi$ quanta are suppressed by the powers of $M^{-1}$.

Although at a first glance the fact that the VEV is larger than the cut-off of the theory might seem a bit unnatural, we should stress that there is nothing unusual in this fact. There exist many well defined examples both in string theory as well as in KK theories when this is the case. After all, the solution of the hierarchy problem in [1] can also be understood in this way. Indeed, the large value of the Planck scale is generated by the size of the extra dimension, or equivalently, by the VEV of the four-dimensional scalar field, the radion, which gets expectation value exceeding by many orders of magnitude the cut-off of the theory.

Another well known example of this kind can be found in $D$-branes. It is well known that in the BPS limit the separation of two parallel $D$ branes can be understood as the Higgs effect in the brane worldvolume gauge theory. The expectation value of the canonically normalized Higgs field is related to the string scale, $M_{S}$, as

$$
<\xi>\sim r M_{S}^{2}
$$

where $r$ is the inter-brane separation. This latter can be much larger than $1 / M_{S}$. In fact the limit $r>>M_{S}^{-1}$ is well defined perturbatively and corresponds to the infrared limit

\footnotetext{
${ }^{5}$ The inflationary solutions in the limit of $\alpha=\infty$ were studied in 21.
} 
for the bulk gravity. Although the world volume scalar field acquires an expectation value which is much larger than the fundamental scale, the theory is well defined. The two-brane system becomes populated with states of mass $\sim r M_{S}^{2}$ that are much heavier than the string scale (corresponding to the stretched string modes in the original theory). It may not be impossible that in a suitable framework like this, the brane separation can set the large coefficient of the brane-induced curvature term.

\section{IMPLICATIONS FOR SUB-MILLIMETER DIMENSIONS}

So far we have been studying the domain $R<r_{c}$. As we have shown, in such a case $R$ can be of astronomical size. In fact, it can be arbitrarily large (if $r_{c}$ is taken to be large). In this section, we shall study the opposite regime $R>r_{c}$. The case $R \rightarrow \infty$ with one extra dimension was already discussed in [3]. In this case the massive KK states which are lighter than $r_{c}$ have unsuppressed wave-functions on the brane. Thus, they couple to the brane-matter with a strength comparable to that of the zero-mode graviton. Therefore, the masses of the KK modes must be large enough in order to avoid unacceptable deviations from Einstein's gravity. Precision measurements of light-bending and the precession of Mercury perihelion exclude the range of masses at the inverse astronomical scale.

The shorter ranges are constrained by the precision measurements of $G$ discussed above. These constraints push $R$ to be somewhere around the inverse millimeter range, in fact, according to recent measurements [19] $M^{-1}>250 \mu$ or so. In such a regime, planetary dynamics is insensitive to extra dimensions, just like in the original scenario of [1]. However, the other predictions of this framework are dramatically modified. The situation is somewhat peculiar for $M^{-1} \ll r_{c} \ll R$. On the one hand, the predictions of [1] for table-top gravitational experiments [20] are unaffected. On the other hand all the current cosmological and astrophysical constraints are lifted, and the collider signatures are dramatically modified. This happens due to the suppression of heavy KK production at high energy colliders.

In order to explicitly demonstrate this let us consider the star cooling process. This process puts the most stringent constraint on the scenario of [1] for the sub-millimeter dimensions. For a given temperature $T$ the production rate of $n$th KK state inside the star is suppressed as

$$
\Gamma \sim \frac{2 T^{3}}{M_{P}^{2}} \sum_{1}^{R T} \frac{1}{1+n^{2}\left(\pi r_{c} / R\right)^{2}}
$$

Assuming that $r_{c} \gg 1 / T$, we can evaluate the contribution of the KK modes with $m<1 / r_{c}$ and $m>1 / r_{c}$ separately and show that these are both of order $\frac{T^{3} R}{M_{P}^{2} r_{c}}$. Using the relation $M_{P}^{3} \sim M^{3} R$ we can bring the total contribution to the form

$$
\Gamma \sim \frac{T^{4}}{M^{3}\left(\operatorname{Tr}_{c}\right)} .
$$

Notice that there is an extra suppression factor $\sim\left(\operatorname{Tr}_{c}\right)$ with respect to the standard case of [1]. This indicates that for sufficiently large $r_{c}$ all the bounds can be avoided. Suppression of the bulk graviton production in other high energy processes can be analyzed in a similar way. The peculiarities of the spectrum of the model indicate that many of the experimental 
constraints on theories with large extra dimensions must be reconsidered in the light of the present discussion.

\section{CONCLUSIONS}

In this paper we studied the scenario of Ref. [3] with graviton kinetic term on the brane with one extra compact dimension. We showed that the existence of the graviton kinetic term on the brane allows for a novel framework with the high-dimensional fundamental Planck scale $M \lesssim \mathrm{TeV}$ (while the particle physics scale is bigger than a $\mathrm{TeV}$ ), and a single flat extra dimension with the size which can be either smaller than $10^{7} \mathrm{~m}$ or larger than $10^{16}$ $\mathrm{m}$.

The crucial role in generating usual 4D Einstein gravity on the brane is played by the brane-induced graviton kinetic term of reference [3]. The strength of this term is governed by a distance scale $r_{c}$ which together with the size of the extra dimension defines the value of the four-dimensional Planck mass (see (1.19)).

There are two phenomenologically interesting regimes. The first one is achieved when $R \ll r_{c} \geq 10^{16} \mathrm{~m}$. In this case the four-dimensional gravity on the brane is mediated by a single non-localized zero mode graviton, both at large and at short distances. The mass spacing of the KK modes is similar to that of an ordinary flat compact dimension. However, the wave-functions of heavy KK modes are suppressed on the brane by the ratio $R / r_{c}$. This gives rise to the effect of "infrared transparency" [1] of the extra space. We studied the constraints imposed by precision gravitational measurements at all scales, as well as restrictions due to various astrophysical and cosmological effects. We found that the model is compatible with all those data. A crucial experimental test of this scenario could arise by observing deviations from Newtonian and Einstein's gravity practically at any scale. This includes precision studies of both relativistic and non-relativistic effects. In the present framework the states that live in the bulk can be a rather exotic source of dark matter. At distances $r \gg R$ their interaction is that of an ordinary dark matter. However, for $r \ll R$ they interact via much stronger gravitational potential which scales as $1 / r^{2}$. Furthermore, these states interact with the observable matter by much weaker gravitational force.

Another interesting limit is $R>r_{c}$ and $N \geq 2$ (in $D=4+N$ dimensional space). In this case the compactification radius $R$ is constrained to be in a sub-millimeter domain. Although this seems to be similar to the scenario of [1], nevertheless, the framework is modified dramatically due to the graviton kinetic term on the brane. For instance, if $M^{-1}<r_{c}<R$, the table-top predictions of scenario [1] are unaffected and one should still expect deviations

from Newton's law at scales $r \sim R$. However, production of heavy KK gravitons is strongly suppressed. This lifts all the high-energy constraints. We have explicitly demonstrated this fact by reevaluating the constraints coming from the process of star cooling. Our analysis demonstrates the crucial importance of the brane-induced graviton kinetic term for the phenomenological studies.

\section{Note added}

After this work was completed, the related work [22] appeared, which discusses the role of brane-induced kinetic term on compact dimensions in an example of a scalar field theory. 


\section{Acknowledgments}

The work of GD was supported in part by David and Lucille Packard Foundation Fellowship for Science and Engineering, by Alfred P. Sloan foundation fellowship and by NSF grant PHY-0070787. The work of GG is supported by DOE Grant DE-FG02-94ER408. The work of MK was supported in part by David and Lucille Packard Foundation. FN is supported by the NYU McCraken Fellowship.

\section{APPENDIX}

In this Appendix we derive the expression for the force mediated by a scalar field in five-dimensional space-time with one compact dimension and an induced kinetic term on a 3-brane. We denote the coordinates $x^{A} \equiv\left(x^{\mu}, y\right), y \in[0, R]$. Our starting point is the following five-dimensional Lagrangian

$$
\mathcal{L}=M^{3} \partial^{A} \Phi \partial_{A} \Phi+\bar{M}^{2} \delta(y) \partial^{\mu} \Phi \partial_{\mu} \Phi,
$$

where $\Phi\left(x^{A}\right)$ has dimension $0, M$ is the fundamental five- dimensional mass scale, and $\bar{M}^{2}$ is the scale which is dynamically generated by the interaction with matter that lives on the brane [3] . If we absorb the factor $M^{3 / 2}$ into the redefinition of the field then $\Phi$ becomes a canonically normalized 5D scalar. The ratio $r_{c}=\bar{M}^{2} / M^{3}$ appears in the field equation as follows:

$$
\partial^{A} \partial_{A} \Phi+r_{c} \delta(y) \partial^{\mu} \partial_{\mu} \Phi=0 .
$$

Let us decompose the field $\Phi$ into the following modes $\Phi=\sum_{m} \phi_{n}(y) \sigma_{n}\left(x^{\mu}\right) . \sigma_{n}\left(x^{\mu}\right)$ satisfy the four dimensional Klein-Gordon equation $\left(\partial^{\mu} \partial_{\mu}+m_{n}^{2}\right) \sigma_{n}=0$, where $m_{n}$ 's are to be determined. The functions $\phi_{n}(y)$ set the profiles of the field in the fifth dimension. From (7.2) (with the use of $\partial^{A} \partial_{A}=\partial^{\mu} \partial_{\mu}-\partial_{y}^{2}$ ) we get the "Schröedinger equation" for $\phi(y)$

$$
\left(\partial_{y}^{2}+m^{2}+r_{c} m^{2} \delta(y)\right) \phi(y)=0 .
$$

This equation is to be considered on a circle of length $R$ with the periodicity condition $\phi(y+R)=\phi(y)$. The problem is equivalent to that of a wave-equation for an infinite space with an array of delta-function type potentials located at $y=n R, n=-0, \pm 1, \pm 2, \ldots$, with the identification $y \equiv y+R$. The procedure for finding the solutions is standard: We solve the equation in two neighboring regions $I \equiv[-R, 0], I I \equiv[0, R]$, match the wavefunction and its derivative at the boundary, and then identify the two regions by imposing the periodicity condition $\phi_{I}(y-R)=\phi_{I I}(y)$. The solutions in each region are

$$
\begin{array}{ll}
\text { I) } & \phi_{I}(y)=A e^{i m y}+B e^{-i m y} \\
\text { II) } & \phi_{I I}(y)=C e^{i m y}+D e^{-i m y}
\end{array}
$$

where the coefficients $A, B, C, D$ are to be determined by the following conditions: (a) periodicity; (b) continuity at $y=0$; (c) matching of first derivatives at $y=0$ : 

a) $A e^{i m(y-R)}+B e^{-i m(y-R)}=C e^{i m y}+D e^{-i m y}$
b) $A+B=C+D$
c) $C-D-A\left(1+i r_{c} m\right)+B\left(1-i r_{c} m\right)=0$.

Solving these algebraic equations we determine the coefficients $B, C, D$ in terms of $A$. This latter can in turn be found from the normalization condition on $\phi$

$$
D=A \quad B=C=A e^{-i m R}
$$

amended by the quantization condition for the masses

$$
-\frac{r_{c}}{R}\left(\frac{m R}{2}\right)=\tan \left(\frac{m R}{2}\right) .
$$

The nonlinear equation $(7.7)$ is solved by graphical method as shown in Fig.2

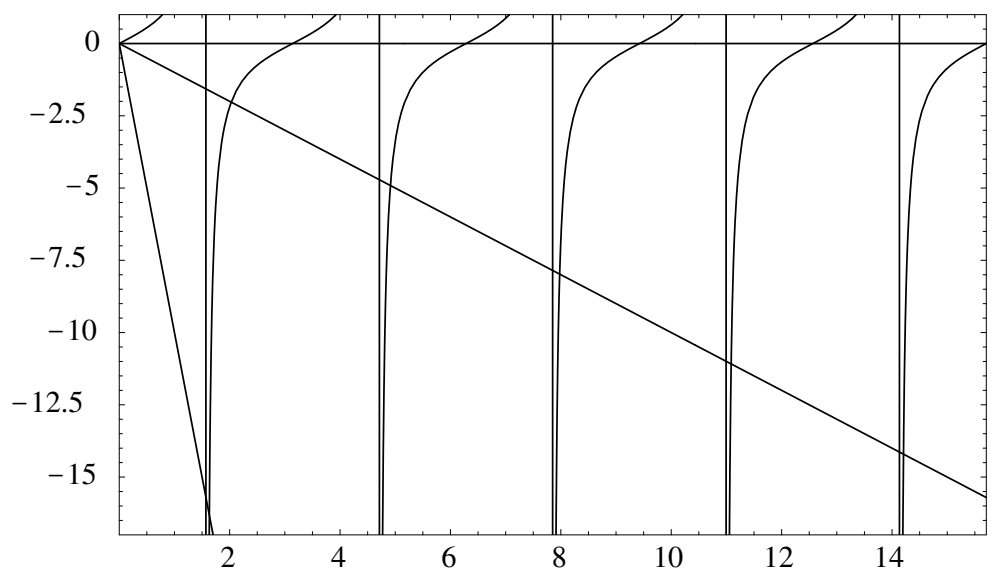

Figure 2: Nonlinear condition for the mass spectrum (7.7). The $x$ axis is given in units of $m R / 2$. The functions shown are tan and the lines with slopes $-r_{c} / R=-10,-1,0$. For $r_{c} / R=0$ the spectrum is standard Kaluza Klein spectrum $m_{n}=2 n \pi / R$, while for large $r_{c} / R$ it is given by $m_{n} \simeq(2 n-1) \pi / R$.

We see that for $r_{c} \ll R$ the masses approach the usual Kaluza-Klein spectrum, $m_{n}=$ $2 \pi n / R$. For $r_{c} \gg R$ all modes (except zero mode) approach the asymptotes of the tangens: $m_{0}=0, m_{n} \simeq(2 n-1) \pi / R$. The level spacing for fixed $R$ does not change. For arbitrary $r_{c}$ the mass of the $n$ 'th state is in the interval $((2 n-1) \pi / R, 2 n \pi / R)$.

Let us now study the behavior of profiles in the fifth dimension. First we have to normalize $\phi_{n}(y)$ to unity. This fixes the coefficient $A_{n}$

$$
\begin{aligned}
& A_{n}=\frac{1}{\sqrt{2 R}} \frac{1}{\sqrt{1-\frac{r_{c} / R}{1+r_{c}^{2} m_{n}^{2} / 4}}} \quad n \neq 0, \\
& A_{0}=\frac{1}{2 \sqrt{R}} .
\end{aligned}
$$

For $r_{c} \gg R$ the coefficient $A$ depends very weakly on the mass of the state and can be approximated by $1 / \sqrt{R}$ for all the modes. In Fig. 3 we show the modulus squared of 
the wave-function for the zero mode (constant) and the lowest three modes for the choice $r_{c} / R=10$.

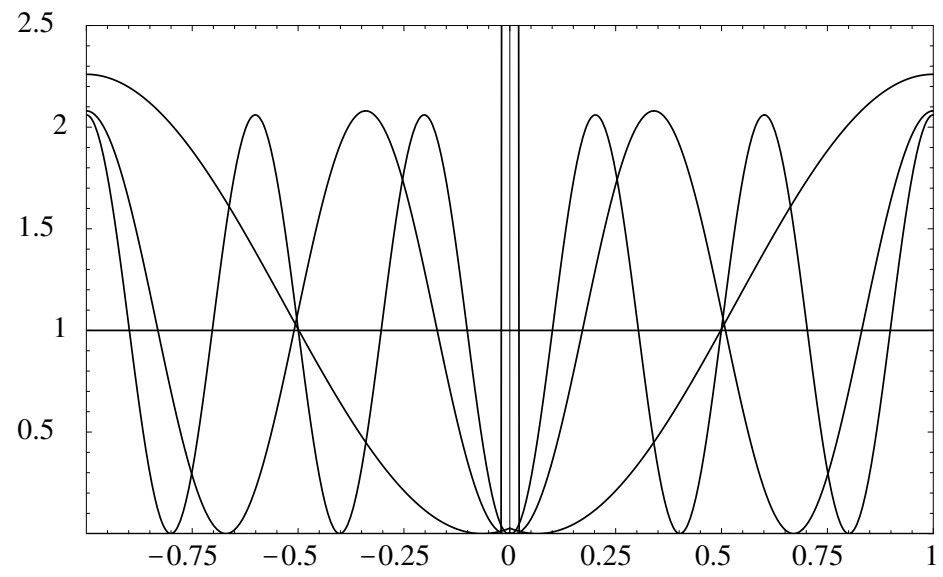

Figure 3: Moduli squared of the wave functions for the zero mode and three lowest modes. The fifth coordinate is shown on the $\mathrm{x}$ axis and the units are $R / 2$ (i.e. the whole range of the extra dimension is covered). The modulus squared is plotted on the y axis and the units are $1 / R$ (i.e. the modulus of the zero mode is equal to one). The dependence is shown for the zero mode (constant), and lowest three modes for $r_{c} / R=10$. The position of the brane at the origin is figuratively sketched as the thin "barrier".

The quantity which determines the coupling of the KK modes to the brane matter is the modulus squared of the wave-function at $y=0$ :

$$
\begin{aligned}
\left|\phi_{n}(0)\right|^{2} & =|A+B|^{2}=|A|^{2}\left|1+e^{-i m_{n} R}\right|^{2} \\
& =|A|^{2} \frac{4}{1+\tan ^{2}\left(m_{n} R / 2\right)}=|A|^{2} \frac{4}{1+r_{c}^{2} m_{n}^{2} / 4} .
\end{aligned}
$$

Here, in the last line we used the condition (7.7). From (7.9) we deduce that higher KK modes are suppressed on the brane compared to the zero-mode. The suppression factor is exactly the same as in theories with infinite volume extra dimension [9]. The mass of the $n$ 'th mode is of order $2 \pi n / R$, so we can rewrite (7.9) in terms of our initial parameters $R, r_{c}$

$$
\frac{\left|\phi_{n}(0)\right|^{2}}{\left|\phi_{0}(0)\right|^{2}} \simeq \frac{1}{1+n^{2}\left(\pi r_{c} / R\right)^{2}} .
$$

For $r_{c} \gg R$ this suppression is substantial even for the lowest massive KK states. In Fig. 4 we show the modulus squared of the three lowest massive modes near the origin for $r_{c} / R=10$. 


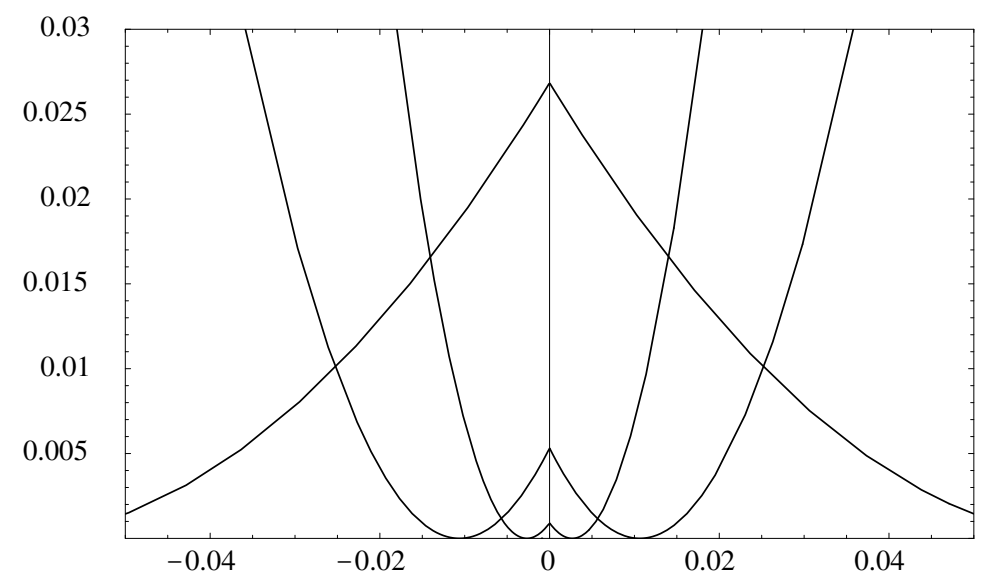

Figure 4: The same as Figure 3. The small portion of space near the origin is shown in order to emphasize the suppression of the first three massive modes on the brane. The zero mode has value one, which is well out of the $\mathrm{y}$ axis range shown in the figure.

Let us now compute the potential due to the exchange of all the modes between two static sources located on the brane. For this purpose we add a source term to (7.1), with a coupling to the field $\Phi$ proportional to $\sqrt{G_{5}}=M^{-3 / 2}$.

Decomposing the five dimensional field in four dimensional modes, $\Phi=\sum_{n} \phi_{n}(y) \sigma_{n}\left(x^{\mu}\right)$, and integrating (7.1) over the extra compact dimension we obtain the following effective four dimensional Lagrangian:

$$
\begin{aligned}
\mathcal{L}_{4}= & \sum_{n, m=0}^{\infty}\left(\partial^{\mu} \sigma_{m} \partial_{\mu} \sigma_{n}\left(\int \mathrm{d} y \phi_{m} \phi_{n}\right)+\left(\sigma_{m} \sigma_{n}\right)\left(\int \mathrm{d} y \partial^{y} \phi_{m} \partial_{y} \phi_{n}\right)\right. \\
& \left.+r_{c}\left(\phi_{m}(0) \phi_{n}(0)\right) \partial^{\mu} \sigma_{m} \partial_{\mu} \sigma_{n}+\delta_{m n} \frac{\phi_{n}(0)}{M^{3 / 2}} \sigma_{n} \rho\right) .
\end{aligned}
$$

Doing the integrals with respect to the compact coordinate $\mathrm{d} y$ one should take into account that the functions $\phi_{n}$ are not orthogonal. Nevertheless, we can proceed as follows: The field equation for $\phi_{m}$ can be written as:

$$
\phi_{m}\left(\partial_{y}^{2}+m_{n}^{2}+r_{c} m_{n}^{2} \delta(y)\right) \phi_{n}=0 .
$$

On the other hand,

$$
\phi_{n}\left(\partial_{y}^{2}+m_{m}^{2}+r_{c} m_{m}^{2} \delta(y)\right) \phi_{m}=0 .
$$

Integrating both equations w.r.t. $y$ and subtracting them, we obtain

$$
0=\left(m_{n}^{2}-m_{m}^{2}\right) \int d y\left(1+r_{c} \delta(y)\right) \phi_{n} \phi_{m} .
$$

This implies that the integral on the right hand side vanishes unless $m=n$. Using this fact, we obtain

$$
\int d y \partial^{y} \phi_{n} \partial_{y} \phi_{m}=m_{n}^{2} \int d y \phi_{n} \phi_{m}\left(1+\delta(y) r_{c}\right)=m_{n}^{2} \delta_{m n}\left(1+r_{c}\left|\phi_{n}(0)\right|^{2}\right) .
$$


Furthermore, inserting (7.15) in (7.11) we get

$$
\mathcal{L}_{4}=\sum_{n=0}^{\infty}\left(\left(1+r_{c}\left|\phi_{n}(0)\right|^{2}\right)\left(\left(\partial^{\mu} \sigma_{n}\right)^{2}+m_{n}^{2}\left(\sigma_{n}\right)^{2}\right)+\frac{\phi_{n}(0)}{M^{3 / 2}} \sigma_{n} \rho\right) .
$$

In order to normalize canonically the kinetic terms we absorb the factor $\left(1+r_{c}\left|\phi_{n}(0)\right|^{2}\right)^{1 / 2}$ into the redefinition of the fields. Thus, the low energy Lagrangian becomes

$$
\mathcal{L}_{4}=\sum_{n=0}^{\infty}\left(\left(\partial^{\mu} \sigma_{n}\right)^{2}+m_{n}^{2}\left(\sigma_{n}\right)^{2}+\frac{1}{\sqrt{1+r_{c}\left|\phi_{n}(0)\right|^{2}}} \frac{\phi_{n}(0)}{\sqrt{M^{3}}} \sigma_{n} \rho\right) .
$$

From $(7.17)$ we can read off the couplings of the various modes to four dimensional matter. In the case of $r_{c} \gg R$, we can approximate $m_{n} \simeq(2 n-1) \pi / R$. Using this and some simplifying algebra we derive:

$$
\begin{aligned}
G_{0} & =\frac{1}{M^{3}} \frac{1}{r_{c}+R} \simeq \frac{1}{r_{c} M^{3}} \equiv G_{\infty}, \\
G_{n} & =\frac{1}{r_{c} M^{3}}\left(\frac{R}{r_{c}}\right) \frac{2}{[(2 n-1) \pi / 2]^{2}+R / r_{c}+\left(R / r_{c}\right)^{2}} \\
& \simeq \frac{1}{r_{c} M^{3}}\left(\frac{2}{\pi^{2}}\right)\left(\frac{R}{r_{c}} \frac{1}{n^{2}}\right) .
\end{aligned}
$$

An equivalent way to extract the information about the potential is to compute the propagator of the field $\Phi$ from the Lagrangian (7.1). For this we have to solve the following equation (for simplicity we work in Euclidean space)

$$
\left[\frac{1}{r_{c}} \partial^{A} \partial_{A}+\delta(y) \partial^{\mu} \partial_{\mu}\right] \Delta(x, y)=-\delta^{4}(x) \delta(y)
$$

where $\Delta$ has dimension two. The procedure is similar to that used in [3] with the only difference being that the fifth dimension is now compact. We turn to 4-dimensional momentum space

$$
\left[\frac{1}{r_{c}}\left(\partial_{y}^{2}-p^{2}\right)-\delta(y) p^{2}\right] \Delta(p, y)=-\delta(y)
$$

and take the ansatz $\Delta(p, y)=D(p, y) B(p)$, with $D(p, y)$ satisfying

$$
\left(\partial_{y}^{2}-p^{2}\right) D(p, y)=-\delta(y)
$$

This gives

$$
B(p)=\frac{r_{c}}{1+p^{2} D(p, 0)}
$$

Eq. (7.22) can be solved in the compact space by expanding both sides in Fourier modes

$$
D(p, y)=\sum_{n=-\infty}^{+\infty} e^{i \frac{2 \pi n}{R} y} D_{n}(p) \quad \delta(y)=\frac{1}{R} \sum_{n=-\infty}^{+\infty} e^{i \frac{2 \pi n}{R} y}
$$


As a result, one finds:

$$
D_{n}=\frac{1}{R} \frac{1}{p^{2}+(2 \pi n / R)^{2}} .
$$

To calculate the force between sources localized on the brane, we need to evaluate the propagator at $y=0$. Using the expression

$$
D(p, 0)=\sum_{n=-\infty}^{+\infty} D_{n}(p)=\frac{1}{p^{2} R}\left[\frac{p R}{2} \operatorname{coth}\left(\frac{p R}{2}\right)\right],
$$

we derive

$$
\begin{aligned}
\Delta(p, 0) & =\frac{1}{p^{2}} \frac{r_{c}}{R} \frac{(p R / 2) \operatorname{coth}(p R / 2)}{1+\left(r_{c} / R\right)(p R / 2) \operatorname{coth}(p R / 2)} \\
& =\frac{1}{p^{2}}\left[\frac{1}{1+\left(1 / p r_{c}\right) 2 \tanh (p R / 2)}\right] .
\end{aligned}
$$

The propagator never deviates substantially from $1 / p^{2}$ over the whole range of $p$. The large- $p$ and small- $p$ behavior are respectively

$$
\begin{array}{ll}
\Delta(p, 0) \simeq \frac{1}{p^{2}}\left[1-\frac{2}{p r_{c}}\right] & p R \gg 1 \text { (short distances) } \\
\Delta(p, 0) \simeq \frac{1}{p^{2}}\left[1-\frac{R}{r_{c}}\right] & p R \ll 1 \text { (large distances). }
\end{array}
$$

The maximal deviation in the coefficient of $1 / p^{2}$ is of order $R / r_{c}$ as before. If we continue this expression to Minkowskian space $p \rightarrow i p_{M}$, the propagator becomes

$$
\Delta(p, 0)=-\frac{1}{p_{M}^{2}}\left[\frac{1}{1+\left(1 / p_{M} r_{c}\right) 2 \tan \left(p_{M} R / 2\right)}\right] .
$$

The poles in this propagator are located at $r_{c} p_{M}=-2 \tan \left(p_{M} R / 2\right)$, in agreement with equation (7.7) . 


\section{REFERENCES}

[1] N. Arkani-Hamed, S. Dimopoulos, G. Dvali, Phys. Lett. B429, 263 (1998);

I. Antoniadis, N. Arkani-Hamed, S. Dimopoulos, G. Dvali, Phys. Lett. B436, 257 (1998).

[2] N. Arkani-Hamed, S. Dimopoulos, G. Dvali, Phys. Rev. D59, 0860 (1999).

[3] G. Dvali, G. Gabadadze and M. Porrati, Phys. Lett. B485, 208 (2000).

[4] G. Dvali and G. Gabadadze, Phys. Rev. D63, 065007 (2001).

[5] A. Vilenkin, Phys. Lett. B133, 177 (1983).

[6] J. Ipser and P. Sikivie, Phys. Rev. D30, 712 (1984);

N. Kaloper and A. Linde, Phys. Rev. D 59, 101303 (1999) hep-th/9811141.

[7] R. Gregory, V.A. Rubakov, S.M. Sibiryakov, Phys. Rev. Lett. 84, 5928 (2000).

[8] C. Csáki, J. Erlich, T.J. Hollowood, Phys. Rev. Lett. 84, 5932 (2000).

[9] G. Dvali, G. Gabadadze, M. Porrati, Phys. Lett. B484, 112 (2000).

[10] G. Dvali, G. Gabadadze, M. Porrati, Phys. Lett. B484, 129 (2000).

[11] L. Pilo, R. Rattazzi, A. Zaffaroni, JHEP 0007, 056 (2000).

[12] C. Csaki, J. Erlich, C. Grojean, T. Hollowood, Nucl.Phys. B584, 359 (2000).

[13] For the generalization to the curved branes, see, M. K. Parikh, S. N. Solodukhin, hepth/0012231.

[14] H. van Dam and M. Veltman, Nucl. Phys. B22, 397 (1970) .

[15] V.I. Zakharov, JETP Lett. 12, 312 (1970) .

[16] C. M. Will, Lecture notes from 26th SLAC Summer Institute on Particle Physics: Gravity - From the Hubble Length to the Planck Length (SSI 98), Stanford, CA, 3-14 Aug 1998. gr-qc/9811036.

[17] C. Talmadge, J.P. Berthias, R.W. Hellings, E.M. Standish, Phys. Rev Lett. 61 (1988), 1159.

[18] E. Fischbach, C. Talmadge, The Search for Non-Newtonian Gravity, Springer-Verlag, 1999.

[19] C. D. Hoyle, U. Schmidt, B. R. Heckel, E. G. Adelberger, J. H. Gundlach, D. J. Kapner and H. E. Swanson, hep-ph/0011014.

[20] J.C. Price, in Proceedings of International Symposium on Experimental Grevitational Physics, ed. Michelson, Guangzhou, China (World Scientific, Singapore,1988);

J. Long, "Laboratory Search for Extra-Dimensional Effectsin Sub-Millimeter Regime" Talk given at the International Conference on Physics Beyond Four Dimensions, ICTP, Trieste, Italy; July 3-6, (2000);

A. Kapitulnik, "Experimental Tests of Gravity Below 1mm" Talk given at the International Conference on Physics Beyond Four Dimensions, ICTP, Trieste, Italy; July 3-6, (2000).

[21] C. Deffayet, hep-th/0010186.

[22] M. Carena, A. Delgado, J. Lykken. S. Pokorski, M. Quiros, C.E.M. Wagner, hep$\mathrm{ph} / 0102172$. 